\section{Reduktion von Trichlormethyl-Verbindungen mit Natriummercaptid + Mercaptan}

Von Friedrich Weygand und Hans Georg Peine

Organisch-chemisches Institut der Technischen Hochschule München

(Z. Naturforschg. 17 b, 205 [1962] ; eingegangen am 29. Januar 1962)

Im Rahmen einer größeren Untersuchung über die Enthalogenierung mit Natriummercaptiden ${ }^{1}$ haben wir auch die Einwirkung von Natriummercaptid oder Natriumthiophenolat in Gegenwart der freien Mercaptane auf einige Trichlormethyl-Verbindungen untersucht. Die bekannte Reaktionsträgheit ${ }^{2}$ von Chloroform, anderen 1.1.1-Trichloralkanen, Benzotrichlorid sowie Tetrachlorkohlenstoff gegenüber Alkalijodid bestätigte sich auch gegenüber den Natriummercaptiden.

In einer kürzlich erschienenen Arbeit beschreibt $\mathrm{KOBER}^{3}$ die Reduktion von Trichlormethyl-s-triazinen und einigen Derivaten oder Trichloressigsäure mit Mercaptanen in Gegenwart von Triäthylamin. Hierbei entstanden die Dichlormethyl-Derivate neben den Mercaptalen.

Wir erhielten aus Trichloressigsäure-methylester in Äther mit Natrium-äthylmercaptid + Äthylmercaptan $77 \%$ an Glyoxylsäure-methylester-diäthylmercaptal und mit Natriumthiophenolat + Thiophenol $51 \%$ an Glyoxylsäure-methylester-diphenyl-mercaptal.

Ferner konnte $\omega$-Tribrom-chinaldin mit Natriumäthylmercaptid + Äthylmercaptan in Äther in 82,5proz. Ausbeute zu Dibrom-chinaldin reduziert werden.

Wie bereits von KовеR gefunden, zeigen auch unsere Ergebnisse, daß das Kohlenstoffatom der Trichlor- oder Tribrommethylgruppe positiviert sein muß, wenn die Reduktion mit Mercaptiden+Mercaptan auf die Aldehydstufe gelingen soll.

\section{Beschreibung der Versuche}

Glyoxylsäure - methylester - diäthylmercaptal: 1,9 g Natrium werden in $200 \mathrm{ccm}$ Äther mit 10,5 g Äthylmercaptan umgesetzt. Beim Rühren über Nacht bildet sich Natriumäthylmercaptid, das sich als farblose Masse absetzt. Bei der allmählichen Zugabe von $15 \mathrm{~g}$ Trichloressigsäure-methylester setzt die Reaktion ohne Wärmezufuhr ein. Bei der Destillation unter Atmosphärendruck nach $5 \mathrm{Stdn}$. gehen zunächst $5,3 \mathrm{~g}$ Diäthyldisulfid über, hierauf isoliert

${ }^{1}$ F. Weygand u. H. G. Peine, Rev. Chim. [Rumänien], im Druck.

2 A. N. Nesmeyanov, R. K. Freidlina u. L. I. Zakharkin, Doklady Akademii Nauk (SSSR) 96, 87 [1954]. man durch Destillation im Vakuum (Sdp..$\left._{11} 122^{\circ}\right) 11,9 \mathrm{~g}$ Glyoxylsäure-methylester-diäthyl-mercaptal.

$\mathrm{C}_{7} \mathrm{H}_{14} \mathrm{O}_{2} \mathrm{~S}_{2}(194,3)$ Ber. C 43,27 H 7,26 Gef. C 43,34 H 7,14.

Zur Identifizierung wurde eine Probe mit 10-proz. methanolischer Kalilauge verseift, das Glyoxylsäuremercaptal mit Brom gespalten ${ }^{4}$ und die Glyoxylsäure als 2.4-Dinitrophenylhydrazon gefällt: Schmp. und Mischschmp. $186^{\circ}$.

Glyoxylsäure-diphenylmercapta l: Zu Natrium-thiophenolat (aus 1,9 g Natrium und 9,5 g Thiophenol) in $200 \mathrm{ccm}$ Äther gibt man 9,5 g Thiophenol und läßt unter Rühren $15 \mathrm{~g}$ Trichloressigsäuremethylester zutropfen. Nach etwa 15 Min. Erhitzen unter Rückfluß setzt die Reaktion ein, worauf noch 5 Stdn. erhitzt wird. Das Natriumchlorid wird abgesaugt und der Äther vertrieben. Der Rückstand wird mit 10-proz. methanolischer Kalilauge verseift. Nach dem Ansäuern mit Schwefelsäure wird in Äther aufgenommen und die Säure mit Natriumhydrogencarbonat zur Abtrennung vom Diphenyldisulfid ausgeschüttelt. Beim Ansäuern der Natriumhydrogencarbonatlösung fällt das Glyoxylsäure-diphenylmercaptal aus, das aus viel Wasser (600 mg aus $1 l$ Wasser) umkristallisiert wird. Ausbeute 11,4 g (51,5\%), Schmp. $104^{\circ}$, federleichte, kleine Kristalle.

$\mathrm{C}_{14} \mathrm{H}_{12} \mathrm{O}_{2} \mathrm{~S}_{2}(276,4)$ Ber. C $60,84 \mathrm{H} \mathrm{4,38} \mathrm{S} \mathrm{23,20.}$

Gef. C 60,76 H 4,43 S 23,10.

Glyox y lsäure-diphen y lmercaptal-di cyclohexylammoniumsalz, Schmp. $126^{\circ}$ (aus Essigester).

$\omega$ - D i brom-chin a $1 \mathrm{~d}$ in : $14 \mathrm{~g} \quad \omega$-Tribromchinaldin werden mit der äquivalenten Menge Natriummercaptid und einem Überschuß von $35 \%$ an Äthylmercaptan in siedendem abs. Äther zur Umsetzung gebracht. Nach einigen Stdn. wird das ausgeschiedene Natriumbromid abfiltriert, worauf das Lösungsmittel und das gebildete Diäthyldisulfid im Vakuum abdestilliert werden. Der Rückstand wird aus Isopropanol umkristallisiert und anschließend sublimiert. Ausbeute $8,4 \mathrm{~g}(82,5 \%)$.

$\mathrm{C}_{10} \mathrm{H}_{7} \mathrm{Br}_{2} \mathrm{~N}(301,1)$ Ber. C 39,90 H 2,35 N 4,65.

Gef. C 39,72 H 2,36 N 4,51.

Zur Charakterisierung wurde eine Probe durch Erhitzen mit Silbernitrat in wäßrigem Alkohol in Chinaldinaldehyd verwandelt, der durch Wasserdampfdestillation nach Versetzen mit Salzsäure sowie Calciumcarbonat isoliert wurde: Schmp. und Mischtemp. $71^{\circ}$ (aus Ligroin).

3 E. Kober, J. org. Chemistry 26, 2270 [1961].

4 F. Weygand u. H. J. Bestmann, Chem. Ber. 89, 1913 [1956]. 